\title{
Microfluidic-based platform to mimic the in vivo peripheral administration of neurotropic nanoparticles
}

\section{Cátia DF Lopes ${ }^{1,2,3}$, Carla $P$ Gomes ${ }^{1,2,}$, Estrela Neto1,2,3, Paula Sampaio1,2, Paulo Aguiar1,2 \& Ana P Pêgo*1,2,4,6}

${ }_{1}$ INEB - Instituto de Engenharia Biomédica, Universidade do Porto, Rua Alfredo Allen, 208, 4200-135 Porto, Portugal.

2i3S - Instituto de Investigação e Inovação em Saúde, Universidade do Porto, Rua Alfredo Allen, 208, 4200-135 Porto, Portugal.

3Faculdade de Medicina da Universidade do Porto, Alameda Prof Hernâni Monteiro, 4200-319 Porto, Portugal.

4Faculdade de Engenharia da Universidade do Porto, Rua Dr Roberto Frias, s/n 4200-465 Porto, Portugal.

5Instituto de Biologia Molecular e Celular, Universidade do Porto, Rua Alfredo Allen, 208, 4200-135 Porto, Portugal.

6 Instituto de Ciências Biomédicas Abel Salazar, Universidade do Porto, Rua de Jorge Viterbo Ferreira, 228, 4050-313 Porto, Portugal.

*Author for correspondence: E-mail Address: apego@ineb.up.pt

Originally published in Nanomedicine (Lond). 2016 Dec;11(24):3205-3221. DOI:10.2217/nnm-20160247 
Keywords: gene delivery $\bullet$ microfluidics $\bullet$ targeted nanoparticles

Aim: Propose a nanoparticle for neuron-targeted retrograde gene delivery and describe a microfluidic-based culture system to provide insight into vector performance and safety. Methods: Using compartmentalized neuron cultures we dissected nanoparticle bioactivity upon delivery taking advantage of (quantitative) bioimaging tools. Results: Targeted and nontargeted nanoparticles were internalized at axon terminals and retrogradely transported to cell bodies at similar average velocities but the former have shown an axonal flux 2.7-times superior to nontargeted nanoparticles, suggesting an improved cargo-transportation efficiency. The peripheral administration of nanoparticles to axon terminals is nontoxic as compared with their direct administration to the cell body or whole neuron. Conclusion: A neuron-targeted nanoparticle system was put forward. Microfluidic-based neuron cultures are proposed as a powerful tool to investigate nanoparticle bioperformance.

The stunning advances of nanotechnology over the past decades have boosted the engineering of biocompatible and biodegradable nanoparticles, allowing their application in the context of human diseases as novel vectors of therapeutic agents. In accordance with the desired purpose, the biophysicochemical properties of nanoparticles such as size, shape, surface charge and surface chemistry can be engineered to tune features that include stability at physiological conditions, encapsulation efficiency and release of therapeutic agents in a controlled manner over time, as well as cellular targeting and uptake efficiency[1].

One area in which the application of nanoparticles is becoming increasingly attractive and may have a significant clinical impact is the neuroscience field. In this context, nanoparticles can be used to mediate the delivery of bioactive molecules within the nervous tissue offering the possibility to reat diseases of the PNS and CNS. In what regards the use of nanoparticles to transfer such bioactive molecules to peripheral neu-rons some distinctive challenges arise. Peripheral neurons are widely distributed through-out the body, with long axons projecting peripherally to the target organs and centrally to the spinal cord. However, their cell bodies are located at the dorsal root ganglia (DRG) or at the spinal cord ventral horn, being relatively inaccessible to direct injection. Besides direct injection (intra ganglionic or intra-spinal) peripheral neuron cell bodies could be reached through intrathecal delivery or through the peripheral administration in the target tissues. From these, the latter prevails as a more clinically relevant route of administration due to its inherent low invasiveness. Thus, in this perspective, a suitable nanoparticle to mediate the delivery of molecules to peripheral neurons upon a peripheral tissue injection should possess the ability to bind specifically to neuron terminals and reach the desired neuron cell bodies by axonal retrograde transport.

Despite the importance of axonal retrograde trans-port for the transference of mitochondria, autophagosomes, lysosomes and growth factors and their receptors to the neuron cell body, it has been demonstrated that the axonal retrograde transport route is also used as the main gateway for the entry and spread of exogenous materials into the nervous system, such as viral agents and neurotoxins (e.g., botulinum or tetanus neurotoxins [TeNT]) [2]. Similarly, nanoparticles can be

INSTITUTO DE INVESTİGAÇÃO E INOVACCÃO EM SAÚDE UNIVERSIDADE DO PORTO

Rua Alfredo Allen, 208 4200-135 Porto Portugal +351220 408800 
designed to mimic the entry of such pathogenic agents and gain access to the peripheral neuron cell bodies through their active retrograde axonal transfer. Thus, understanding how nanoparticles' biophysicochemical properties relate to their uptake and retrograde transport along axons is an important step toward the developing of effective nano-based therapies.

Due to the lack of resolution and sensitivity, in vivo imaging techniques (See [3] for an Editorial) do not allow the real-time tracking of nanoparticle-loaded vesicles in individual axons. Alternatively, the direct visualization of nanoparticle retrograde axonal trans-port and its quantitative analysis have been evaluated in in vitro neuronal cultures[4, 5], but the use of conventional cultures, where the nanoparticles are placed in contact with the whole neuron, hampers the out-come translation to in vivo conditions. Under these conditions, neurons can uptake nanoparticles along all cell plasma membrane (cell body, neurites, axons and axon terminals), and the anterograde and retrograde nanoparticle trafficking processes can occur simultaneously. A reliable in vitro assay for live imaging evaluation of nanoparticle retrograde axonal trans-port should recapitulate the desired in vivo peripheral route of administration in target tissues, in which nanoparticles only contact the neuron axon terminals. The recent introduction of microfluidic chambers as a tool in the context of neuronal cultures has allowed the establishment of compartmentalized cultures where neuron cell bodies and axonal terminals are physically separated. These microfluidic chambers are com-posed of two or more compartments interconnected by microgrooves that assure the separation of cell bodies from axonal terminals, thus, allowing precise spatial control of experimental conditions where axonal terminals and cell bodies can be subjected to selective treatments. The use of such microfluidic systems has been increasingly applied to neurosciences namely to study neuron/nonneuronal cells or tissue interactions $[6,7]$, axonal growth[8, 9], axonal mRNA transcripts [10], axonal motor proteins [11], axonal transport of neuro-trophins like NGF [12] and BDNF [13], tau proteins [14], neurotoxins $[15]$, virus $[16,17]$ and mitochondria [18].

In this study we explore the use of a microfluidic-based DRG neuron culture to test the uptake and transport kinetics of gene carrying trimethylated chitosan (TMC)-based nanoparticles actively targeted to neurons by surface functionalization with the non-toxic and neurotropic C-terminal 54 $\mathrm{kDa}$ fragment of the TeNT heavy chain (HC) [19], while mimicking a peripheral in vivo route of administration. This approach proved to be suitable to confirm, supported by real-time nanoparticleloaded vesicle visualization and quantification, the active retrograde transport of nanoparticles. To the best of our knowledge, this is the first report showing the applicability of such in vitro microfluidicbased platforms as a model to simulate a peripheral route of administration for neurotropic nanoparticles and evaluate their uptake, retrograde axonal transport and toxicity profile in peripheral nervous system neurons.

\section{Materials \& methods}

\section{Preparation of microfluidic chambers for primary neuronal cultures}

The microfluidic chambers were prepared by placing the microfluidic devices (AXIS450, Millipore, France) against a glass coverslip. Briefly, sterile square glass coverslips $(24 \times 24 \mathrm{~mm}$, Microscopic Glass Factory [MGF]-slides, Immuno-Cell, Belgium) were coated with $0.01 \mathrm{mg} / \mathrm{ml}$ of poly(D-lysine) (PDL, MW $30-70 \mathrm{kDa}$, Sigma-Aldrich Co., MO, USA) overnight at $37^{\circ} \mathrm{C}$, washed with Milli- ${ }^{\circledR}$ water (Millipore) and completely air-dried under sterile conditions. Microfluidic devices were sterilized with 
$70 \%$ ethanol for 5 min as indicated by the manufacturer. After completely air-dried microfluidic devices were gently attached to PDL-coated glass coverslips, creating a microfluidic chamber composed of two compartments separated from each other by $450 \mu \mathrm{m}$ length $\times 5 \mu \mathrm{m}$ height $\times 10 \mu \mathrm{m}$ width microgrooves. The medium reservoirs were loaded with $150 \mu \mathrm{l}$ of $5 \mu \mathrm{g} / \mathrm{ml}$ laminin-1 isolated from mouse Engelbreth-Holm-Swarm sarcoma (Sigma-Aldrich Co.) and incubated overnight at $37^{\circ} \mathrm{C}$. The unbounded laminin-1 was removed and chambers were washed once with DMEM with GlutaMAX ${ }^{\mathrm{TM}}$ (Gibco ${ }^{\circledR}$, Thermo Fisher Scientific, MA, USA) refilled with DMEM medium and let to equilibrate for at least $2 \mathrm{~h}$ at $37^{\circ} \mathrm{C}$, prior to cell seeding.

\section{Primary cultures of DRG neurons}

All experiments using animals were carried out with the permission of the local animal ethical committee in accordance with the EU Directive (2010/63/EU) and Portuguese law ( $D L$ 113/2013). The experimental protocol (reference 421/000/000/2013) was approved by the ethics committee of the Portuguese official authority on animal welfare and experimentation (Direção-Geral de Alimentação e Veterinária).Primary embryonic rat DRG neurons were isolated from Wistar embryo rats (E18). Briefly, rat embryos were dissected in Hank's buffer solution (Sigma-Aldrich Co.) and enzymatically treated with $1.25 \%(\mathrm{w} / \mathrm{v})$ collagenase type II (Gibco, Thermo Fisher Scientific) at $37^{\circ} \mathrm{C}$ for $90 \mathrm{~min}$. Subsequently, collagenase was removed and DRGs washed three-times in DMEM followed by sequential mechanical dissociation with 1000 and $200 \mu$ l pipette tips. Viable cells were counted using the trypan blue (0.4\% [w/v], Sigma-Aldrich Co.) exclusion assay and seeded at a density of $1 \times 107$ viable cells $/ \mathrm{ml}$. For the microfluidic-based cultures, $5 \mu$ l of the cell suspension was pipetted to the cell body compartment (Figure $1 \mathrm{~A}$ ). For conventional cultures, $5 \times 104$ cells $/ \mathrm{cm} 2$ were seeded in 48 -well plates (for internalization assay) on glass cover-slips (10 mm, MGF-slides) or $1.56 \times 105$ cells/cm2 were seeded in 96-well plates (for metabolic activity assay), both previously treated with $0.01 \mathrm{mg} / \mathrm{ml}$ PDL as described above. Cells were allowed to attach for $1 \mathrm{~h}$ at $37^{\circ} \mathrm{C}$. Subsequently, complete neuron culture medium constituted by DMEM/Ham's $\mathrm{F}_{12}$ with GlutaMAX (DMEM/F12), $50 \mu \mathrm{g} / \mathrm{ml}$ of penicillin and streptomycin, $25 \mathrm{ug} / \mathrm{ml}$ fungizone, $2 \%$ (v/v) B27 supplement (all from Gibco, Thermo Fisher Scientific) and $50 \mathrm{ng} / \mathrm{ml}$ of NGF $7 \mathrm{~S}$ (Calbiochem ${ }^{\circledR}$, Millipore) was added to each cell culture platform. The culture medium was replaced every third day and cultures were kept in a humidified incubator at $37^{\circ} \mathrm{C}$ supplied with $5 \% \mathrm{CO}_{2}$. This DRG neuron isolation protocol resulted in $72 \%$ of neuron purity at $48 \mathrm{~h}$ postseeding, determined by the percentage of neurons (immunostained for $\beta$ III tubulin, a neuron-specific microtubule marker using the monoclonal antibody mouse anti- $\beta$ III tubulin $[1: 1,000$; Promega, WI, USA]) in relation to the total number of cells in culture (identified by nuclei staining with $0.1 \mu \mathrm{g} / \mathrm{ml}_{\text {4', }}$-diamidino-2-phenylindole, Sigma-Aldrich Co.).

\section{Preparation of rhodamine-labeled TMC-based nanoparticles}

\section{Synthesis of partially thiolated TMC}

TMC derived from ultrapure chitosan produced from Agaricus bisporus mushrooms, under current Good Manufacturing Practice standards, (40 kDa, KitoZyme, Belgium) was purified by filtration and dialysis prior to use, as described previously [20]. In brief, TMC was diluted in $5 \mathrm{mM} \mathrm{HCl}$ solution at a final concentration of $0.5 \%(\mathrm{w} / \mathrm{v})$, filtered through a Buchner funnel and purified by dialysis using a 3.5 kDa MW cut-off membrane (Spectrum Labs, CA, USA) for 3 days against deionized water and collected after freeze-drying. The degree of acetylation and quaternization of the purified TMC was characterized by $1 \mathrm{H}$-nuclear magnetic resonance spectroscopy and determined to be $15.7 \pm 0.9 \%$ and 
$30.1 \pm 4.6 \%$, respectively. Endotoxin levels of the purified polymer extracts were assessed using the Limulus Amebocyte Lysate Assay (QCL-1000, Cambrex, NJ, USA), following the manufacturer instructions. Endotoxin levels were found to be $<0.1 \mathrm{EU} \mathrm{ml} / 1$ (an EU correspond-ing to a unit of measurement for endotoxin activity), respecting the US Department of Health and Human Services guidelines for implantable devices [21]. After freeze-dried, TMC was dissolved in $20 \mathrm{mM} \mathrm{HEPES}$ (Sigma-Aldrich Co)-buffered saline solution containing $5 \%(\mathrm{w} / \mathrm{v})$ glucose $(\mathrm{pH} 7.4)$ at a $0.25 \%(\mathrm{w} / \mathrm{v})$ final concentration. Purified TMC was then partially thiolated by the immobilization of thiol groups from 2-iminothiolane on the primary amino groups of the polymer, as described elsewhere [22]. Briefly, TMC was dissolved in $20 \mathrm{mM}$ HEPES buffer at $5 \mathrm{mg} / \mathrm{ml}$ and 2 -iminothiolane hydrochloride (SigmaAldrich Co.) was added to the polymer solution, in order to attain a theoretical modification of $10 \%$ of the polymer primary amine groups. Afterward, the $\mathrm{pH}$ of the solution was adjusted to 8 with $\mathrm{NaOH}$. After $6 \mathrm{~h}$ of incubation at room temperature (RT), under an inert atmosphere saturated with argon and with continuous stirring, the resulting thiolated TMC (TMCSH) was dialyzed for 3 days against 5 $\mathrm{mM} \mathrm{HCl}$ at $4^{\circ} \mathrm{C}$. Thereafter, samples were frozen at $-80^{\circ} \mathrm{C}$ and lyophilized for 3 days. The resulting powders were stored at $-20^{\circ} \mathrm{C}$ until further use.

To allow the tracking of TMCSH-based nanoparticles, the polymer was fluorescently labeled with the rhodamine-activated derivative 5(6)-carboxy-X-rhodamine $\mathrm{N}$-succinimidyl ester (ROX) (Sigma-

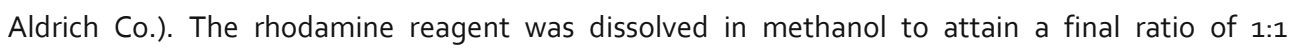

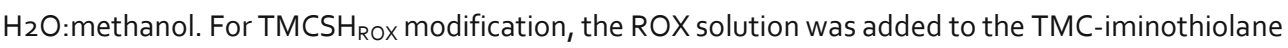
hydrochloride reaction, after $3 \mathrm{~h}$ of incubation, in a $\mathrm{NH}_{2}$ : $\mathrm{ROX}$ ratio of 7.4:1. The reaction was then incubated for an additional period of $3 \mathrm{~h}$, in an inert atmosphere at RT and under continuous stirring. The resulting polymer conjugate was then purified as described above.

\section{Determination of polymer thiol content}

The degree of polymer thiolation was quantified with 5,5-dithio-bis-(2-nitrobenzoic acid) (DTNB, Ellman's reagent). The amount of free thiol groups grafted to the polymer was determined based in a pre-viously described protocol [19] and found to be $149.1 \pm 15.8 \mu \mathrm{mol} / \mathrm{g}$, corresponding to $4.7 \pm 0.5 \%$ substitution of TMC primary amines by thiol groups. Briefly,

DTNB was dissolved in $0.1 \mathrm{M}$ sodium phosphate buf-fer, $\mathrm{pH} 8$ at a final concentration of $100 \mu \mathrm{g} / \mathrm{ml}$. The polymer samples were dissolved in the same solution at a final concentration of $2.5 \mathrm{mg} / \mathrm{ml}$. Then, $20 \mu \mathrm{l}$ of the polymer solution was mixed with $180 \mu \mathrm{l}$ of DTNB reagent solution in a microplate and after incubation of $15 \mathrm{~min}$ at RT the absorbance was measured at a wave-length of $405 \mathrm{~nm}$ (Spectra Max GeminiXS, Molecu-lar Devices, CA, USA). Cysteine standards were used to calculate the amount of free thiol moieties in the polymer.

\section{Plasmid DNA production \& purification}

The plasmid DNA (pDNA) used encoded for the TdTomato protein (pCSCMV:tdTomato, $5.5 \mathrm{~kb}$, Addgene, UK) and was produced in $\mathrm{DH}_{5} \alpha$ competent Escherichia coli strain and purified using a EndoFree plasmid Giga kit (Qiagen, Germany) following the manufacturer's instructions. Plasmid concentration and purity were assessed by UV spectroscopy (Nano-drop ND-1000, Thermo Fisher Scientific) and the absorbance ratio achieved $(260 / 280 \mathrm{~nm})$ was between 1.8 and 2.0.

\section{HC fragment production, purification \& modification}


The plasmid encoding for HC fragment was a kind offer from Neil Fairweather (King's College, UK). The pro-duction using transformed BL21 Escherichia coli strain and purification of $\mathrm{HC}$ fragment was performed as pre-viously described[19]. The purified fragment was then modified with a bifunctional 5 kDa PEG spacer (JenKem Technology, China) bearing an $\mathrm{N}$-hydroxysuccinimide and a maleimide end group, at a $2.5 \mathrm{PEG} / \mathrm{HC}$ protein molar ratio. The amount of reactive maleimide groups in the $\mathrm{HC}$ fragment was determined using a modified Ellman's assay [23] and found to be $1.5 \mathrm{~mol}$ PEG/HC

\title{
Nanoparticle preparation
}

TMCSH-based nanoparticles were prepared using an N/P molar ratio (moles of quaternized amine groups [N] to moles of DNA phosphate groups [P]) of 8. Briefly, the nanoparticle core was formed by mixing, while vortexing, equal volumes of plasmid DNA solu-tion with TMCSH solution in $20 \mathrm{mM}$ HEPES-buff-ered saline solution containing $5 \%(\mathrm{w} / \mathrm{v})$ glucose, $\mathrm{pH} 7.4$. Complexes were let to stabilize for $15 \mathrm{~min}$ at RT and then the pegylated HC fragment (HC-PEG) was added to the nanoparticle solution in a w/w ratio of HC-PEG per pDNA of 4 , and let to react for $24 \mathrm{~h}$ under agitation, at RT.

\section{Rhodamine labeling of HC fragment}

$\mathrm{HC}$ fragment alone was used as a control in the cellular uptake studies. To allow the tracking of $\mathrm{HC}$ fragment it was fluorescently labeled with ROX. In brief, $0.4 \mathrm{ml}$ of rhodamine solution $(2.5 \mathrm{mg} / \mathrm{ml}$ in dehydrated dimethylformamide) was added in drop wise to $6 \mathrm{mg}$ of purified HC protein at $1 \mathrm{mg} / \mathrm{ml}$ in $0.1 \mathrm{M}$ phosphate buffer ( $\mathrm{pH}$ 5.5) and let to react for 1 h, at RT, under constant stirring and protected from light. The purified rhodamine-labeled $\mathrm{HC}$ fragment (HCROX) was washed several times with 0.1 $\mathrm{M}$ phosphate buffer and concentrated using a $30 \mathrm{kDa}$ cut-off filter (Amicon Ultra, Mil-lipore). Subsequently, $\mathrm{HCROX}$ solution was filtered and stored at $-20^{\circ} \mathrm{C}$ until further use. Characterization of TMCSH-based nanoparticlesTMCSH-based nanoparticles grafted or not with HC-PEG were characterized in terms of size, polydispersity index ( $\mathrm{Pdi}$ ) and $\zeta$ potential using a Zetasizer Nano Zs (Malvern, UK). The Smoluchowski model was applied for $\zeta$ potential determination and cumulants analysis was used for $Z$-average particle size determination. Ten micrograms of pDNA were used to prepare the tested formulations. All measurements were performed in triplicate, at $25^{\circ} \mathrm{C}$.

\section{Cellular uptake of TMC-based nanoparticles}

To evaluate the cellular interaction and uptake of non-targeted TMCSHRox and neurotropic $\mathrm{TMCSH}_{\mathrm{ROX}}-\mathrm{HC}$ nanoparticles we performed an internalization assay using the microfluidic and conventional-dissociated DRG neuron cultures. The $H C_{R O X}$ fragment was used as control. For microfluidic-based cultures $\mathrm{HC}_{\mathrm{ROX}}$ or nanoparticles were supplied in the medium of the axonal compartment while for conventional cultures a similar amount of $H C_{R O X}$ or nanoparticles were provided to the whole culture in the culture medium (Figure $1 B$ \& $C$, respectively). Particular care was taken in the microfluidic-based cultures to assure that the media volume at the cell body compartment was at all times higher than the media volume at the axonal compartment. This small hydrostatic pressure difference resulted in a slow but continuous flow of medium across the microgrooves from the somal side to the axonal side, preventing the free passage of $\mathrm{HC}$ protein or nanoparticles to the cell body compartment. Following an incubation of 5 or $12 \mathrm{~h}$, at $37^{\circ} \mathrm{C}$, unbounded $\mathrm{HC}_{\mathrm{RO}}$ protein or nanoparticles were washed two-times with phosphate-buffered saline and cells were fixed with $4 \%(\mathrm{w} / \mathrm{v})$ paraformaldehyde. To discriminate neurons from the nonneuronal cells present in culture, fixed cells were stained as previously described.

\author{
(n)
}




\section{Microfluidic live imaging \& analysis Nanoparticle transport}

Live imaging was performed at $37^{\circ} \mathrm{C}$ using a laser scanning confocal microscope Leica TCS SP 5 II (Leica Microsystems, Germany) with the HC PL APO CS $40 \times / 1.10$ water or HC PL APO Lbl. Blue $63 \times / 1.40$ oil objectives. Laser line at $561 \mathrm{~nm}$ was used for rhodamine excitation. For each treatment group, a total of 24 microgrooves (containing at least one axon) were randomly selected for live imaging. The microgrooves were imaged in the proximity of the cell body compartment, $1 \mathrm{~h}$ following TMC-based nanoparticles application in the axonal compartment. All image series were acquired for 3-5 min, $4 \mathrm{~s} /$ frame. This interval of seconds between frames allowed for long-term imaging without dye bleaching. Images were acquired from three independent experiments. For the tracking and analysis of nanoparticle-loaded vesicles $\left(\mathrm{TMCSH}_{\mathrm{ROx}}\right.$ positive vesicles), 12 images series for each condition were selected. Images series were analyzed using a custom-made program written in MATLAB (The MathWorks, version 2015a, MA, USA). The program provided tools for semiautomatic and manual tracking of the vesicles, and a tool for the calculation of the flux of vesicles in the axons.

For tracking, image series were preprocessed with a Laplacian of Gaussian filter scaled according to the median size of the nanoparticle-loaded vesicles. This provided an enhancement of blob-like structures in the image and, as a consequence, an increase in contrast between vesicles and background. Segmentation was performed through a user-defined intensity threshold. The center of each vesicle was calculated as the centroid in the intensity profile. Semiautomated tracking was performed whenever possible - in these cases the user selected the seed locations of vesicles and a detection-association algorithm, based on near-est neighbor detections between consecutive frames, automatically followed the vesicles trajectories. The trajectories were reconstructed manually in situations where the vesicles contrast was low or the vesicles positional changes between frames were large enough to impair the detection-association algorithm (meaning that semiautomated tracking was not feasible). For the analysis, only moving vesicles tracked over at least four consecutive frames within a sequence of frames were considered. Vesicles that moved further than $10 \mu \mathrm{m}$ were classified as a run. Any movement $<10 \mu \mathrm{m}$ (between two pauses or between the beginning or end of the sequence and a pause) was not considered as a run and was integrated into the adjacent pause period. A pause was determined as a sequence of frames during which the vesicles moved below the instantaneous speed of $0.1 \mu \mathrm{m} / \mathrm{s}$ in each frame, with a minimum duration of $8 \mathrm{~s}$. The instantaneous velocity was determined by the ratio between the distance $(\mu \mathrm{m})$ and the time (s) covered by a vesicle in two consecutive frames. Using these criteria, the probability density function of nanoparticle-loaded vesicle instantaneous velocity, the average velocity of each vesicle, the length of each run and the duration of each pause were quantified.

The axonal flux of nanoparticle-loaded vesicles in the retrograde direction was quantified based on the velocity and density of vesicles (number and size of vesicles) passing through the axon's crosssection. It is presented as the number/density of particles per unit of length (the axon's cross section) per unit of time. To estimate the flux of nanoparticles in the image series, it was assumed that the amount of nanoparticles in a vesicle is proportional to the total intensity level (fluorescence) of the area occupied by the vesicle (in the image). Furthermore, flux for a specific experimental condition was estimated in terms of mean values: the mean vesicle density was multiplied by the mean vesicles velocity to give the mean flux for the specific experimental condition. Mean vesicle density was calculated by image analysis in a sequence of steps. First, image series were preprocessed cropping the images to the microgroove interior area and performing noise reduction using a Gaussian filter with a spatial scale smaller than the average vesicle size. Segmentation was then performed and the 
background was zeroed. The sum of all positive pixels was then taken from the difference between consecutive frames. This operation allowed for the selective integration of intensities associated with moving structures. The vesicle density, for each pair of images, was given by this sum, normalized by the total area of the image (cropped to the microgroove borders). Mean values and standard deviations were obtained from the full sequence in the image series. Mean vesicles velocity was calculated from the tracking of the vesicles trajectory. The velocity of each vesicle was calculated performing a linear regression on the axonal axial position as a function of time.

\section{Mitochondria motility assessment after nanoparticle treatment}

The axonal transport of mitochondria was analyzed by confocal live imaging to determine if nanoparticle treatment impacts the overall mitochondria motility. Briefly, mitochondria of DRG neurons cultured in microfluidic chambers were labeled with $200 \mathrm{nM}$ Mitotracker ${ }^{\circledR}$ Green FM (Molecular Probes, Thermo Fisher Scientific) for $20 \mathrm{~min}$ at $37^{\circ} \mathrm{C}$. Afterward, cells were washed once with medium and kept in a humidi-fied incubator overnight, at $37^{\circ} \mathrm{C}$ supplied with $5 \% \mathrm{CO}_{2}$. Nanoparticles were then supplied in the axonal compartment and incubated with axon terminals for $1 \mathrm{~h}$ at $37^{\circ} \mathrm{C}$. Subsequently, axons within microgrooves were live imaged using a laser scanning confocal microscope Leica TCS SP 5 II (Leica Microsystems, Germany) with the HC PL APO Lbl. Blue $63 \times / 1.40$ oil objective. Laser line at $488 \mathrm{~nm}$ was used for Alexa Fluor ${ }^{\circledR} 488$ excitation (Thermo Fisher Scientific). For each treatment group, a total of 12 microgrooves (containing at least one axon) were randomly selected for mitochondria tracking. All image series were acquired for $3 \mathrm{~min}, 1 \mathrm{~s} /$ frame. Images were acquired from three independent experiments. Images series were analyzed using a custom-made program written in MATLAB. For each condition, imaged mitochondria were characterized into moving or paused/stationary mitochondria. The average velocity $(\mu \mathrm{m} / \mathrm{s})$ of moving mitochondria, either showing retrograde, anterograde or bidirectional movement, was additionally determined for each treatment condition.

\section{Intracellular vesicle labeling}

DRG neurons treated with both nanoparticle for-mulations were immunostained for the clathrin and caveolin-1 proteins, structural components of the intracellular vesicles, to check for co-localization with nanoparticles. In brief, after $1 \mathrm{~h}$ of nanoparticles incubation into the axonal compartment of microfluidic cultures, unbounded rhodamine-labeled nanoparticles were washed two-times with phosphate-buffered saline and cells were fixed with $4 \%(\mathrm{w} / \mathrm{v})$ paraformaldehyde. Afterward, clathrinand caveolin-1-associated vesicles were immunostained using a monoclonal anti-body rabbit anticlathrin (1:50; Cell Signaling Technology, MA, USA) or a monoclonal antibody rabbit anti-caveolin-1 (1:200; Cell Signaling Technology). A polyclonal goat anti-rabbit Alexa Fluor 488 conjugated (1:500; Molecular Probes $^{\mathrm{TM}}$, Thermo Fisher Scientific) was used as secondary antibody.

\section{Neuronal metabolic activity assay}

The neuronal metabolic activity was assessed, after incubation of both culture types with the nanoparticles, by measuring the adenosine 5 -triphosphate (ATP) levels. The nanoparticles vehicle (20 mM HEPES-buffered saline solution containing $5 \%(\mathrm{w} / \mathrm{v}$ ) glucose $[\mathrm{pH} 7.4]$ ), TMCSH or TMCSH-HC nanoparticles were added to the whole medium in conventional cultures or to the cell body or axonal compartment medium of microfluidic cultures, and let in contact for 6 or $12 \mathrm{~h}$. Intracellular ATP levels were measured using the CellTiter-Glo ${ }^{\circledR}$ Luminescent Cell Viability Assay (Promega) with some

\section{.}


adaptations from the manufacturer's protocol. In brief, cells in 96-well plate and microfluidic chambers were gently trypsinized, collected and washed with medium. The CellTiter-Glo ${ }^{\circledR}$ Reagent was added to the cells resulting in cell lysis and generation of a luminescent signal proportional to the amount of cellular ATP present in each condition. As recommended by the manufacturer, the luminescence was measured after $10 \mathrm{~min}$ of incubation in a luminescent plate reader (SYNERGY MX, BioTeK, France). ATP disodium salt hydrate (Sigma-Aldrich Co.) was used to prepare an ATP standard curve.

\section{Statistical analysis}

Statistical analysis was performed using GraphPad Prism version 5.0 for Windows (CA, USA). Unless mentioned otherwise, results are presented as mean \pm standard deviation. The D'Agostino and Pearson omnibus normality test was used to test if data obeyed to a Gaussian distribution. To verify whether ROX-labeling interfered with the physicochemical properties of nanoparticles, the nonparametric Mann-Whitney $U$ test was performed to compare each nanoparticle formulation before and after ROX labeling. To deter-mine whether differences existed between treatments regarding duration of pause periods and axonal flux, an unpaired t-test was used. As the average velocity of each vesicle and run length parameters did not follow a Gaussian distribution the nonparametric Mann-Whitney $U$ test was performed to compare both treatment groups. To determine whether a difference in mitochondria axonal transport and ATP synthesis existed between nanoparticle treatment and control, in each culture condition, the one-way ANOVA test was performed followed by the Tukey's post-test. Results with $p<0.05$ were considered statistically significant.

\section{Results}

\section{Nanoparticle physicochemical characterization}

Both nanoparticle formulations used in the present study - nontargeted $\mathrm{TMCSH}_{\mathrm{ROx}}$ and neurotropic $\mathrm{TMCSH}_{\mathrm{ROX}}-\mathrm{HC}$ nanoparticles - were characterized at first in terms of size, Pdi and $\zeta$ potential. As illustrated in Table 1, the mean size diameter values obtained for $\mathrm{TMCSH}_{\mathrm{ROX}}-\mathrm{HC}$ nanoparticles is not significantly different from the naked nanoparticles showing that $\mathrm{HC}$ functionalization does not influence the average size of the nanoparticles. $\zeta$ potential values obtained for both nanoparticles were positive and not significantly different, indicating a cationic surface charge of nanoparticles with a good colloidal stability, independent of HC functionalization. Moreover, it was confirmed that the size and $\zeta$ potential values of ROX-labeled nanoparticles were not significantly different from their unlabeled counterparts (Supplementary Table 1 ) indicating that the nanoparticle-labeling procedure does not influence their main physicochemical properties.

\section{Cellular uptake of TMC-based nanoparticles}

Dissociated DRG neuron cultures were incubated with nanoparticles for 5 or $12 \mathrm{~h}$, at $37^{\circ} \mathrm{C}$. In conventional DRG neuron cultures, $\mathrm{HC}_{\mathrm{ROX}}$ or nanoparticles were supplied in the culture medium to the whole culture (Figure ${ }_{1} C$ ), while in microfluidic-based cultures these were supplied only in the culture medium of the axonal compartment (Figure $1 \mathrm{~B}$ ). Confocal microscopy analysis demonstrated a widespread cellular internalization of $\mathrm{HC}_{\mathrm{ROX}}, \mathrm{TMCSH}_{\mathrm{ROX}}$ and $\mathrm{TMCSH}_{\mathrm{ROX}}-\mathrm{HC}$ in conventional cultures (Figure 2 for $5 \mathrm{~h}$ and Supplementary Figure 1 for $12 \mathrm{~h}$ of incubation), regard-less of the incubation

(1) 
period or cellular type. Furthermore, a direct relation between incubation time and cellular internalization extent was not observed. In microfluidic DRG neuron cultures, HCRox and both nanoparticle formulations were highly associated with axonal terminals in the axonal compartment (Figure ${ }_{3} G, H$ \& I) and were found in some neurons at the cell body compartment, although in different quantities concerning each treatment condition and time of incubation. After $5 \mathrm{~h}$ of incubation, $\mathrm{HC}_{\mathrm{ROX}}$ was detected within the cell body of neurons that exclusively lay in the cell body compartment of the microfluidic chambers, enclosed in small round shape structures, suggesting it has been condensed in intra-cellular vesicles that were found distributed the cytoplasm (Figure $3 \mathrm{~A}$ ). Contrarily, TMCSH $\mathrm{ROX}_{\mathrm{HC}} \mathrm{HC}$ nanoparticles, although also detected at the micro-fluidic cell body compartment, were only detected in few axonal processes (Figure ${ }_{3} C$ ), while evidence of $\mathrm{TMCSH}_{\text {ROX }}$ nanoparticles in this compartment was not noticeable at this time point of analysis. The increase in incubation time resulted in a higher accumulation of $H C_{R O x}$ in the neuron cell bodies that was detected in vesicles predominantly located at the nuclei periphery (Figure $3 \mathrm{D}$ ). Similarly, after $12 \mathrm{~h}$ of incubation, $\mathrm{TMCSH}_{\mathrm{ROX}}$ and $\mathrm{TMCSH} \mathrm{ROX}_{\mathrm{R}} \mathrm{HC}$ nanoparticles were detected in neuron cell bodies in small round vesicular structures, being the amount of $\mathrm{TMCSH}_{\mathrm{ROX}}-\mathrm{HC}$ nanoparticle-loaded vesicles higher than the ones carrying the $\mathrm{TMCSH}_{\mathrm{ROx}}$ nanoparticles (Figure $3 \mathrm{E} \& \mathrm{~F}$, respectively).

Another important finding observed in microfluidic DRG neuron cultures was the fact that $\mathrm{HC}_{\mathrm{ROx}}$ or nanoparticles were not detected in all neuron cell bodies neither in any of the non-neuronal cells present at the cell body compartment, confirming that only the neurons that had grown their axons to the axonal compartment were able to internalize and transport the $\mathrm{HC}_{\mathrm{ROx}}$ or nanoparticles to their cell bodies.

\section{TMCSH-based nanoparticles retrograde axonal transport}

To explore the ability of TMCSH-based nanoparticles to be retrogradely transported from axonal terminals to the neuron cell bodies, live imaging was per-formed using the microfluidic DRG neuron cultures. Nanoparticles were supplied to the medium in the axonal compartment and axons inside microgrooves were subsequently imaged in the proximity of the cell body compartment.

Live imaging analysis showed several nanoparticle-loaded vesicles being retrogradely transported in axons after internalization in their terminals at the axonal compartment (Figure 4 \& Supplementary Videos 1 \& 2). $\mathrm{TMCSH}_{\mathrm{ROX}}-\mathrm{HC}$ nanoparticle-loaded vesicles were observed in retrograde axonal transit in all axons imaged. Contrarily, not all axons imaged for $\mathrm{TMCSH}_{\mathrm{ROx}}$ nanoparticles showed the transport of nanoparticle-loaded vesicles (only 75\%), and the total amount of transported nanoparticles in each axon appears to be lower than the one observed for $\mathrm{TMCSH}_{\mathrm{ROX}}-\mathrm{HC}$ nanoparticles (Supplementary Videos 1 \& 2). Immunostaining of vesicles being transported along the observed axons revealed several co-localization events of both nanoparticle formulations with clathrin and caveolin-1-associated vesicles, although these were more frequent for clathrin than caveolin-1-positive structures in the case of $\mathrm{TMCSH}_{\mathrm{ROX}}-\mathrm{HC}$ (Supplementary Figure 2).

By monitoring and tracking the nanoparticle trafficking along the axons, the motion properties of loaded vesicles with both nanoparticle types were characterized using a number of parameters, namely the probability density function of the instantaneous velocity, the average velocity, the time spent in movement versus pause and the axonal flux. The probability density function of instantaneous velocity showed more variability and higher median instantaneous velocity for $\mathrm{TMCSH}_{\mathrm{ROX}}$-loaded vesicles as compared with the peaked $\mathrm{TMCSH}_{\mathrm{ROX}}-\mathrm{HC}$-loaded vesicles velocity 
distribution (Figure $5 \mathrm{~A}$ ). Also, vesicles carrying $\mathrm{TMCSH}_{\mathrm{ROx}}$ nanoparticles showed more anterograde movements (depicted by negative values of the instantaneous velocity) that represented short reversals in direction for vesicles that were moving in the net retro-grade direction. However, when looking into the aver-age velocity values, vesicles loaded with $\mathrm{TMCSH}_{\mathrm{ROX}}$ or $\mathrm{TMCSH}_{\mathrm{ROX}}-\mathrm{HC}$ nanoparticles were transported both at high velocities $(>1 \mu \mathrm{m} / \mathrm{s})$, in the range of fast axonal transport (Figure ${ }_{5} \mathrm{~B}$ ), made long runs with similar total distances progressed (Figure ${ }_{5} \mathrm{C}$ ) and spent similar pause time periods during a run (Figure $5 \mathrm{D}$ ). Nevertheless, the axonal flux of nanoparticle-loaded vesicles in axonal transit was significantly higher for the ones carrying the $\mathrm{TMCSH}_{\mathrm{ROX}}-\mathrm{HC}$ nanoparticles (Figure ${ }_{5} \mathrm{~F}$ ). As described before, the physical quantity flux takes concurrently into consideration the density of vesicles (number and size of vesicles) and their velocity (Figure $5 \mathrm{E}$ ). This assessment is relevant as axons with nanoparticle-loaded vesicles travelling all at the same velocity may present distinct fluxes if the density of vesicles is different (either due to the fact that the vesicles have distinct sizes, or are present in different numbers, as illustrated in Figure $5 \mathrm{E}$ axons $\mathrm{A}$ and $\mathrm{B}$ ). Conversely, axons can present similar fluxes, even when the transported vesicles are present in similar densities, when the vesicles are being transported at different average velocities (Figure $5 \mathrm{E}-$ axon $\mathrm{A}$ and $\mathrm{C}$ ).

\section{Mitochondria axonal transport}

To determine if nanoparticle treatment modifies the normal axonal transport in neurons we evaluated and compared the axonal transport of mitochondria between control (untreated) and nanoparticle-treated neuron cultures (after $1 \mathrm{~h}$ of contact with the nanoparticle formulations) (Supplementary Videos 3-5). Our results show that the majority of axonal mitochondria remain paused/stationary during the acquisition time (approximately $80 \%$ ), both in control and nanoparticletreated neurons (Figure 6A). Moreover, in all groups in study the moving mitochondria presented a similar average transport velocity (Figure 6B).

\section{Neuronal metabolic activity}

To compare the impact of nanoparticle administration to the whole cell versus to the axonal terminals (as occurs in an in vivo peripheral administration in target tissues), we assessed the cellular metabolic activity by measuring the intracellular ATP levels after 6 and $12 \mathrm{~h}$ of nanoparticle incubation in conventional and micro-fluidic cultures, respectively. To discard any interference that could result from the use of different cell culture conditions (conventional versus microfluidic), we also compared the impact of nanoparticle administration directly to the cell bodies versus the axonal terminals in a microfluidic setup. Cells cultured in conventional platforms demonstrated a sharp decrease in their intra-cellular ATP levels for both nanoparticle formulations, compared with untreated cells (Figure 7). Conversely, cells cultured in microfluidic chambers and treated with nanoparticles in the axonal compartment, either maintained their intracellular ATP levels similar to the control (Figure 7) or presented a significant increase in their intracellular ATP levels as observed at $6 \mathrm{~h}$ post-treatment with the TMCSH nanoparticles ( Figure 7A). Additionally, cells cultured in microfluidic chambers and treated with nanoparticles in the cell body versus the axonal compartment showed a similar behavior, with significantly reduced ATP levels being observed when nanoparticles were administrated directly to the cell bodies (Figure $7 C \& D$ ).

\section{Discussion}

西 
Aiming at developing new therapeutic strategies to treat nervous system diseases, researchers have been exploring the development of targeted nanoparticles to mediate the delivery of therapeutic molecules to specific neuron populations, as well as clinical relevant routes for heir administration in the body. Our research group is particularly interested in the development of biocompatible and biodegradable nanoparticles to mediate the targeted delivery of therapeutic genes to DRG neurons upon their peripheral and minimally invasive administration in target tissues. This route of administration requires that the selected nanoparticles have to be internalized at the axon terminals and be retrogradely transported to the neuron cell body. To attain nanoparticle neurotropism we are exploring the tethering of the nanoparticles' surface with a pegylated HC fragment, as previously reported[19], since this nontoxic fragment of the TeNT is described to be neurotropic and capable of being retrogradely transported along peripheral neu-rons[24, 25]. Nevertheless, the assessment of retrograde axonal transport ability of these nanoparticles has been hampered by the limited imaging resolution and sensitivity inherent to currently available in vivo real-time imaging techniques, as well as the inefficacy of conventional in vitro neuronal cultures to mimic a peripheral in vivo administration for these nanoparticles. These shortcomings motivated us to establish an in vitro plat-form that can recapitulate an in vivo peripheral route of nanoparticle administration by using a microfluidic chamber to culture dissociated DRG neurons.

The use of microfluidic chambers offers technical advantages over conventional cultures. The embedded microgrooves in the microfluidic chamber allow axons to grow through it and to be efficiently isolated in the axonal compartment. Moreover, in such platforms one can restrict nanoparticle internalization to occur only at axon terminals, which is not possible to control in conventional cultures. Furthermore, in this manner, a large number of axons can be analyzed in a given chamber allowing the axonal transport evaluation and quantification. Our results on the study of cellular uptake of nanoparticles corroborate the advantage of using microfluidic neuron cultures over conventional ones. DRG neuron conventional cultures incubated with the two-tested nanoparticle formulations (targeted versus nontargeted) resulted in a broad cellular nanoparticle uptake, despite cellular type and nanoparticle formulation. In opposition, using microfluidic DRG neuron cultures, different nanoparticle accumulation rates in the neuron cell body were observed for the $\mathrm{TMCSH}_{\mathrm{ROX}}$ and $\mathrm{TMCSH}_{\mathrm{ROX}}-\mathrm{HC}$ formulations, suggesting a different uptake mechanism for each nanoparticle type. These findings highlight the limited utility of conventional cultures to discriminate such differences for nanoparticles with distinct surface functionalization.

Furthermore, in these microfluidic platforms, one was able to explore, using confocal live cell imaging, the uptake and axonal transport kinetics of TMC-SHROX and $\mathrm{TMCSH}_{\mathrm{ROX}}-\mathrm{HC}$ nanoparticles upon their contact with axonal terminals. Both nanoparticle formulations were found to be transported with similar average velocities, in the range of fast axonal transport $[26,27]$. This observation suggests that nanoparticle surface functionalization with the $\mathrm{HC}$ fragment does not influence the retrograde axonal transport average velocity of these nanoparticles. Rather, both naked and $\mathrm{HC}$-functionalized nanoparticles may be transported in vesicles directed to the neuron cell body using the microtubule-dependent molecular motor dynein, the responsible for cargoes retrograde axonal transport [28]. However, our data for the probability density function of the instantaneous velocity showed a different distribution of the instantaneous velocities for vesicles carrying each nanoparticle formulation. $\mathrm{TMCSH}_{\mathrm{ROX}}-\mathrm{HC}$ nanoparticle-loaded vesicles showed a picked instantaneous velocity distribution ranging from 0.2 to $3.9 \mu \mathrm{m} / \mathrm{s}$, similar to what has been described for retrograde axonal transport of HC fragment of TeNT in vitro[29, 30]. In contrast, $\mathrm{TMCSH}_{\mathrm{ROX}}$ nanoparticle-loaded vesicles displayed a variable instantaneous velocity distribution and also some 
movements in the anterograde direction. This change in $\mathrm{TMCSH}_{\mathrm{ROx}}$ nanoparticle-loaded vesicles motility probably arises from changes in the number of motor proteins actively attached to these vesicles at a particular time that may be influenced by their cargo, as has been argued in previous studies [31, 32

While the type of intracellular transport (passive versus active) involved in nanoparticle trafficking can be assessed based on velocity studies, the efficiency of a nanoparticle in targeting the soma is more accurately captured by the flux of loaded vesicles in the retrograde transport, as opposed to considering uniquely the velocity. Here the nanoparticle axonal flux was quantified taking into consideration the velocity as well as the density of vesicles (number and size) passing through an axon's cross-section. Our results disclosed a higher axonal flux for vesicles carrying the TMCSH ROX$\mathrm{HC}$ nanoparticles, evidencing a more efficient retrograde axonal transport of these vesicles as compared with the vesicles transporting the $\mathrm{TMCSH}_{\mathrm{ROx}}$ nanoparticles. Taking into account that the average velocity of both nanoparticle formulations is similar, this difference can only be explained by a different density of vesicles in axonal transit for each condition that could be dependent on differences in vesicle number, size or both.

Taken together, these observations suggest that the process of binding/internalization for each nanoparticle formulation is distinct and this may result in different efficiencies in the retrograde axonal transport associated with each nanoparticle formulation. We hypothesize that $\mathrm{TMCSH}_{\mathrm{ROX}}-\mathrm{HC}$ interact with axon plasma membrane mainly through TeNT receptor complex, constituted by polysialogangliosides and glycosylphosphatidylinositol-anchored proteins [33, 34], and are internalized as a consequence of membrane receptor activation in clathrin-mediated endosomes, as has been reported for HC [33]. Immunostaining of vesicles being transported along the observed axons revealed a higher occurrence of co-localization of $\mathrm{TMCSH}_{\mathrm{ROX}}-\mathrm{HC}$ nanoparticles and clathrinpositive vesicles than caveolin-1-positive structures (Supplementary Figure 2). This receptormediated internalization and transport hypothesis is in line with our previous observations with HCfunctionalized nanoparticles based either on poly(ethylene imine) [19, 35] or chitosan [36]. Once inside the cell, vesicles carrying $\mathrm{TMCSH}_{\mathrm{ROX}}-\mathrm{HC}$ nanoparticles interact with dynein/dynactin complex and are retrogradely transported to the cell body, with an average velocity similar to what has been reported to the fast retrograde axonal transport. In opposition, $\mathrm{TMCSH}_{\mathrm{ROx}}$ nanoparticles interact with the axon plasma membrane through electrostatic interactions. Although $\mathrm{TMCSH}_{\mathrm{ROX}}$ nanoparticles were found to co-localize with clathrin and caveolin-1-associated vesicles (Supplementary Figure 2), we believe that the inexistence of a targeting moiety in these nanoparticles favors a nonspecific internalization, possibly in nonclathrin and noncaveolae-mediated endosomes [37]. Previous reports have proven that sulfated proteoglycans present at the cellular plasma membrane contribute, together with phospholipids, to its negative net charge and can act as plasma membrane carriers [38] and mediate cellular internalization of cationic delivery systems [39, 40]. This nonspecific internalization process in the context of the axonal terminals is probably not as efficient as the receptor-mediated uptake, which can explain the fact that not all axons imaged in the microfluidic chambers transported vesicles containing the TMC-SHRox nanoparticles. Nevertheless, upon internalization vesicles transporting the $\mathrm{TMCSH}_{\mathrm{ROx}}$ nanoparticles that interact with the dynein/dynactin complex are retrogradely transported to the cell body as well.

Since nanoparticle toxicity at the cellular level can translate into clinical toxicity, we took advantage of these microfluidic DRG neuron cultures to explore the impact of nanoparticle administration on neuron metabolic activity. Our results demonstrate that both nanoparticle formulations exerted a 
marked drop in metabolic activity as compared with control when in contact with the cell body or whole cell (conventional cultures) rather than in contact with the axonal terminals. This can be related to higher interactions with cell membrane, internalization events and intracellular accumulation observed in cell body and conventional administration conditions. In opposition, the administration of nanoparticles solely in the proximity of the axonal terminals did not affect the cellular metabolic activity, being the ATP consumption levels equal to the control. Moreover, we show that nanoparticle treatment does not influence the mitochondria axonal transport, at least at this time point of analysis. It has been previously reported that mitochondria alter their motility under certain stress conditions or when their integrity is impaired [41]. Based on the above one can conclude that different nanoparticle routes of administration can trigger distinctive biological responses for the same nanoparticle formulation. This highlights the importance of conducting cytotoxicity studies in similar conditions to their desired route of administration in the body, in detriment of eliminating useful nanoparticle formulations.

\section{Conclusion}

Here we describe the use of a microfluidic-based DRG neuron culture to mimic in vitro an in vivo peripheral route of nanoparticle administration near axonal terminals (e.g. intramuscular delivery). Using this platform we were able to discriminate the trafficking properties of different nanoparticle formulations, such as the higher axonal flux of TMCSH-HC nanoparticles-loaded vesicles, otherwise not feasible using con-ventional DRG neuron cultures, inferring from the efficiency of the delivery process mediated by different nanoparticles. Moreover, this system showed to be of added value in the evaluation of nanoparticle-mediated cytotoxic effects, an issue that can be extended to other research areas to allow the study of other nanoparticle formulations safety. The application of this micro-fluidic-based platform provided experimental evidence for the retrograde axonal transport and safety of TMCSH-HC nanoparticles that can be applied to subsequent in vivo studies. Finally, this work further contributes to an emerging literature demonstrating the advantageous application of microfluidic devices for neuroscience-related research.

Altogether our results show that this microfluidic-based neuron culture is a powerful tool to investigate nanoparticle bio-performance, namely, regarding cell-nanoparticle interactions, nanoparticle axonal trans-port and safety, allowing for their rapid translation to subsequent in vivo studies.

\section{Acknowledgements}

The authors acknowledge the Biointerfaces and Nanotechnology Service of Instituto de Investigação e Inovação em Saúde for the nanoparticle characterization studies and the Centro de Materiais da Universidade do Porto (CEMUP) for nuclear magnetic resonance analysis 


\section{Financial \& competing interests disclosure}

The work was financed by Portuguese funds through the FCT - Fundação para a Ciência e a Tecnologia in the frame -work of the projects PTDC/CTM-NAN/115124/2009 and PTDC/CTMNAN/3547/2014. CDF Lopes, CP Gomes and E Neto acknowledge the FCT for their PhD scholarships (SFRH/BD/77933/2011, SFRH/BD/77930/2011 and SFRH/BD/81152/2011, respectively). The authors have no other relevant affiliations or financial involvement with any organization or entity with a financial interest in or financial conflict with the subject matter or materials discussed in the manuscript apart from those disclosed.

No writing assistance was utilized in the production of this manuscrip

\section{Ethical conduct of research}

The authors state that they have obtained appropriate institu -tional review board approval or have followed the principles outlined in the Declaration of Helsinki for all human or animal experimental investigations. In addition, for investigations in-volving human subjects, informed consent has been obtainedfrom the participants involve 


\section{REFERENCES}

$1 \mathrm{Nel} A E$, Madler L, Velegol $D$ et al. Understanding biophysicochemical interactions at the nano-bio interface. Nat. Mater. 8(7), 543-557 (2009). • Highlights the main properties of nanoparticles that shape their interaction with cells/tissue and that could determine their biocompatibility/safety.

2 Salinas S, Schiavo G, Kremer EJ. A hitchhiker's guide to the nervous system: the complex journey of viruses and toxins. Nat. Rev. Microbiol. 8(9), 645-655 (2010). • Highlights the strategies used by several pathogens (viruses and bacterial toxins) to access and spread into the nervous system upon their interaction with membrane receptors present at the nerve terminals.

3 Lopes CDF, Gomez-Lazaro M, Pêgo AP. Seeing is believing but quantifying is deciding. Nanomedicine 10(15), 2307-2310 (2015). • Authors highlight the many challenges that remain ahead in the understanding of nanoparticle intracellular fate, mainly by questioning the relevance of conventional in vitro models to predict the in vivo nanoparticle behavior.

4 Steketee MB, Moysidis SN, Jin XL et al. Nanoparticle-mediated signaling endosome localization regulates growth cone motility and neurite growth. Proc. Natl. Acad. Sci. USA 108(47), 19042-19047 (2011).

5 Wong Y, Markham K, Xu ZP et al. Efficient delivery of siRNA to cortical neurons using layered double hydroxide nanoparticles. Biomaterials 31(33), 8770-8779 (2010).

6 Neto E, Alves CJ, Sousa DM et al. Sensory neurons and osteoblasts: close partners in a microfluidic platform. Integr. Biol. (Camb.) 6(6), 586-595 (2014).

7 Pagella P, Neto E, Jimenez-Rojo L, Lamghari M, Mitsiadis TA. Microfluidics co-culture systems for studying tooth innervation. Front. Physiol. 5, 326 (2014).

8 Hengst U, Deglincerti A, Kim HJ, Jeon NL, Jaffrey SR. Axonal elongation triggered by stimulus-induced local translation of a polarity complex protein. Nat. Cell Biol. 11(8), 1024-1030 (2009).

9 Millet LJ, Stewart ME, Nuzzo RG, Gillette MU. Guiding neuron development with planar surface gradients of substrate cues deposited using microfluidic devices. Lab Chip 10(12), 1525-1535 (2010).

10 Taylor AM, Berchtold NC, Perreau VM, Tu CH, Li Jeon N, Cotman CW. Axonal mRNA in uninjured and regenerating cortical mammalian axons. J. Neurosci. 29(15), 4697-4707 (2009).

11 Chowdary PD, Che DL, Kaplan L et al. Nanoparticle-assisted optical tethering of endosomes reveals the cooperative function of dyneins in retrograde axonal transport. Sci. Rep. 5, 18059 (2015).

12 Zhang K, Osakada Y, Vrljic M, Chen L, Mudrakola HV, Cui B. Single-molecule imaging of NGF axonal transport in microfluidic devices. Lab Chip 10(19), 2566-2573 (2010).

13 Poon WW, Blurton-Jones $\mathrm{M}$, Tu CH et al. $\beta$-amyloid impairs axonal BDNF retrograde trafficking. Neurobiol. Aging 32(5), 821-833 (2011).

14 Stoothoff W, Jones PB, Spires-Jones TL et al. Differential effect of three-repeat and four-repeat tau on mitochondrial axonal transport. J. Neurochem. 111(2), 417-427 (2009).

15 Wang T, Martin S, Papadopulos A et al. Control of autophagosome axonal retrograde flux by presynaptic activity unveiled using botulinum neurotoxin type A. J. Neurosci. 35(15), 6179-6194 (2015).

16 Feiler MS, Strobel B, Freischmidt A et al. TDP-43 is intercellularly transmitted across axon terminals. J. Cell Biol. 211(4), 897-911 (2015)

17 Castle MJ, Perlson E, Holzbaur EL, Wolfe JH. Long-distance axonal transport of AAVg is driven by dynein and kinesin-2 and is trafficked in a highly motile Rab7-positive compartment. Mol. Ther. 22(3), 554-566 (2014). 
$18 \mathrm{Kim} \mathrm{HJ}$, Park JW, Byun JH et al. Quantitative analysis of axonal transport by using compartmentalized and surface micropatterned culture of neurons. ACS Chem. Neurosci. 3(6), 433-438 (2012).

19 Oliveira H, Fernandez R, Pires LR et al. Targeted gene delivery into peripheral sensorial neurons mediated by self-assembled vectors composed of poly(ethylene imine) and tetanus toxin fragment c. J. Control. Release 143(3), 350-358 (2010). • The first report describing the functionalization of thiolated poly(ethylene imine)-based nanoparticles with a pegylated HC fragment and their biophysicochemical properties.

20 Moreno PM, Santos JC, Gomes CP et al. Delivery of splice switching oligonucleotides by amphiphilic chitosanbased nanoparticles. Mol. Pharm. 13(2), 344-356 (2016).

21 Administration USFDA. Guidance for industry - pyrogen and endotoxins testing (2012). www.fda.gov/drugs/guidancecomplianceregulatoryinformation/guidances/ucm314718.htm

22 Verheul RJ, Van Der Wal S, Hennink WE. Tailorable thiolated trimethyl chitosans for covalently stabilized nanoparticles. Biomacromolecules 11(8), 1965-1971 (2010).

23 Ellman GL. Tissue sulfhydryl groups. Arch. Biochem. Biophys. 82(1), 70-77 (1959).

24 Herreros J, Lalli G, Schiavo G. C-terminal half of tetanus toxin fragment $C$ is sufficient for neuronal binding and interaction with a putative protein receptor. Biochem. J. 347(Pt 1), 199-204 (2000).

25 Fishman PS, Carrigan DR. Retrograde transneuronal transfer of the C-fragment of tetanus toxin. Brain Res. 406(1-2), 275-279 (1987).

26 Grafstein B, Forman DS. Intracellular transport in neurons. Physiol. Rev. 6o(4), 1167-1283 (1980).

27 Brown A. Axonal transport of membranous and nonmembranous cargoes: a unified perspective. J. Cell Biol. $160(6), 817-821$ (2003).

28 Roberts AJ, Kon T, Knight PJ, Sutoh K, Burgess SA. Functions and mechanics of dynein motor proteins. Nat. Rev. Mol. Cell Biol. 14(11), 713-726 (2013). • Offers an overview of the functions and mechanism of action of dynein, the motor protein responsible for mediating the retrograde transport.

29 Lalli G, Schiavo G. Analysis of retrograde transport in motor neurons reveals common endocytic carriers for tetanus toxin and neurotrophin receptor p75NTR. J. Cell Biol. 156(2), 233-239 (2002).

30 Lalli G, Gschmeissner S, Schiavo G. Myosin Va and microtubule-based motors are required for fast axonal retrograde transport of tetanus toxin in motor neurons. J. Cell Sci. 116(Pt 22), 4639-4650 (2003).

31 Hill DB, Plaza MJ, Bonin K, Holzwarth G. Fast vesicle transport in PC12 neurites: velocities and forces. Eur. Biophys. J. 33(7), 623-632 (2004).

32 Hendricks AG, Perlson E, Ross JL, Schroeder HW 3rd, Tokito M, Holzbaur EL. Motor coordination via a tug-ofwar mechanism drives bidirectional vesicle transport. Curr. Biol. 20(8), 697-702 (2010).

33 Deinhardt K, Berninghausen O, Willison HJ, Hopkins CR, Schiavo G. Tetanus toxin is internalized by a sequential clathrin-dependent mechanism initiated within lipid microdomains and independent of epsin1. J. Cell Biol. 174(3), 459-471 (2006).

34 Lalli G, Bohnert S, Deinhardt K, Verastegui C, Schiavo G. The journey of tetanus and botulinum neurotoxins in neurons. Trends Microbiol. 11(9), 431-437 (2003).

35 Oliveira H, Rangl M, Ebner A, Mayer B, Hinterdorfer P, Pego AP. Molecular recognition force spectroscopy: a new tool to tailor targeted nanoparticles. Small 7(9), 1236-1241 (2011).

36 Oliveira H, Pires LR, Fernandez R, Martins MC, Simoes S, Pego AP. Chitosan-based gene delivery vectors targeted to the peripheral nervous system. J. Biomed. Mater. Res. A 95(3), 801-810 (2010). • Describes the functionalization and biophysicochemical characterization of chitosan-based nanoparticles with the pegylated $\mathrm{HC}$ fragment.

INSTITUTO DE INVESTIIGAÇÃO E INOVAÇÃO EM SAÚDE UNIVERSIDADE DO PORTO

Rua Alfredo Allen, 208 4200-135 Porto Portugal +351220408800 
37 Perumal OP, Inapagolla R, Kannan S, Kannan RM. The effect of surface functionality on cellular trafficking of dendrimers. Biomaterials 29(24-25), 3469-3476 (2008).

38 Belting M. Heparan sulfate proteoglycan as a plasma membrane carrier. Trends Biochem. Sci. 28(3), 145-151 (2003).

39 Mislick KA, Baldeschwieler JD. Evidence for the role of proteoglycans in cation-mediated gene transfer. Proc. Natl. Acad. Sci. USA 93(22), 12349-12354 (1996).

40 Mounkes LC, Zhong W, Cipres-Palacin G, Heath TD, Debs RJ. Proteoglycans mediate cationic liposome-DNA complex-based gene delivery in vitro and in vivo. J. Biol. Chem. 273(40), 26164-26170 (1998).

41 Sheng ZH. Mitochondrial trafficking and anchoring in neurons: new insight and implications. J. Cell Biol. 204(7), 1087-1098 (2014). 


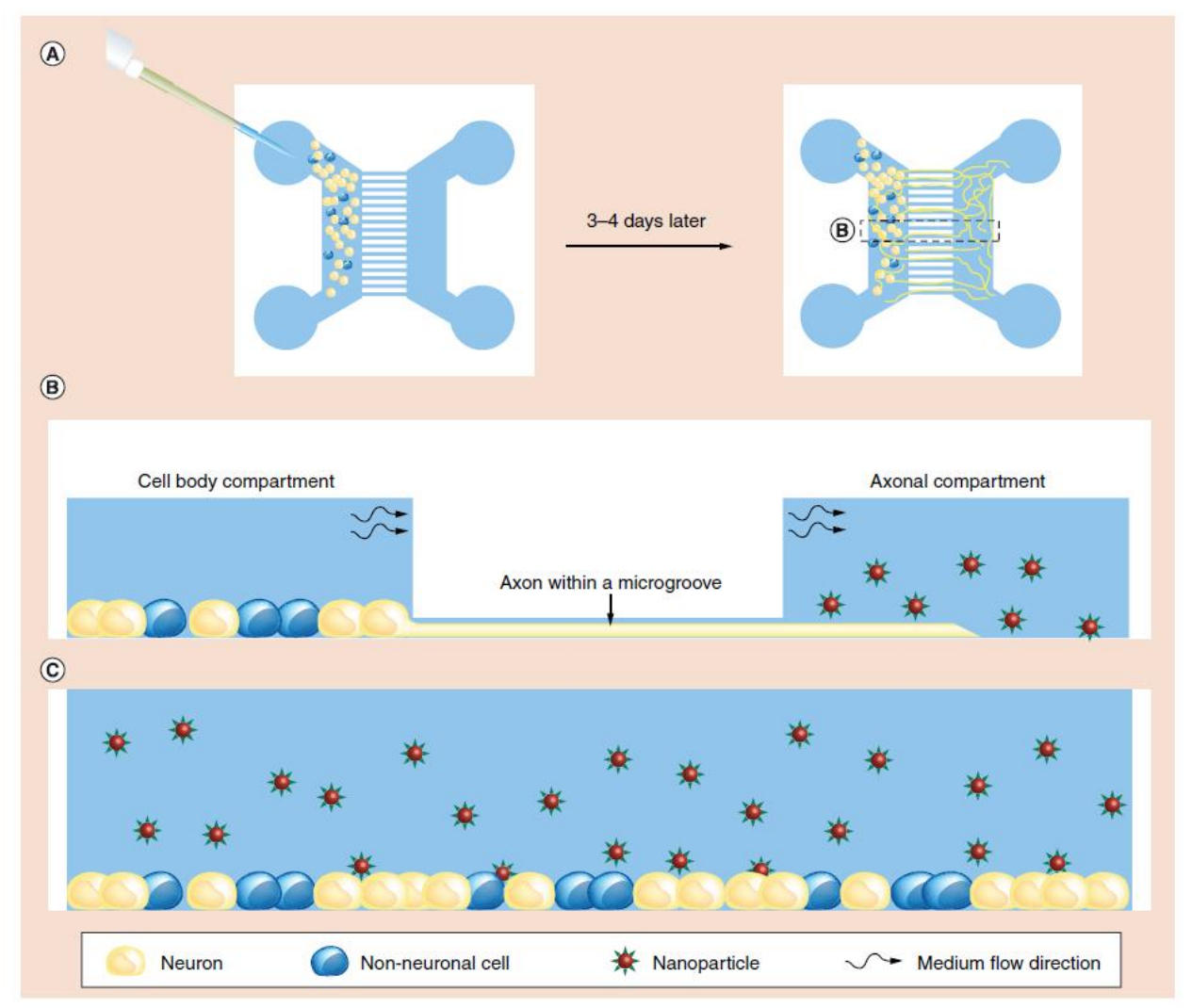

Figure 1. Schematic representation of microfluidic-based and conventional dorsal root ganglia neuron cultures. (A) Illustration of cell seeding in the cell body compartment of microfluidic chambers. Neurons grow their axons to the axonal compartment in 3-4 days after seeding. (B) Side-view illustration of nanoparticle incubation in microfluidic or (C) conventional DRG neuron cultures. In microfluidic-based cultures, nanoparticles are supplied in the culture media in the axonal compartment while in conventional cultures nanoparticles are supplied in culture media to the whole culture. In microfluidic-based cultures, a difference in media volume was used to induce a small hydrostatic pressure variance and force the media flow across the microgrooves from the cell body compartment to the axonal compartment, avoiding the free passage of nanoparticles to the cell body compartment.

DRG: Dorsal root ganglia. 

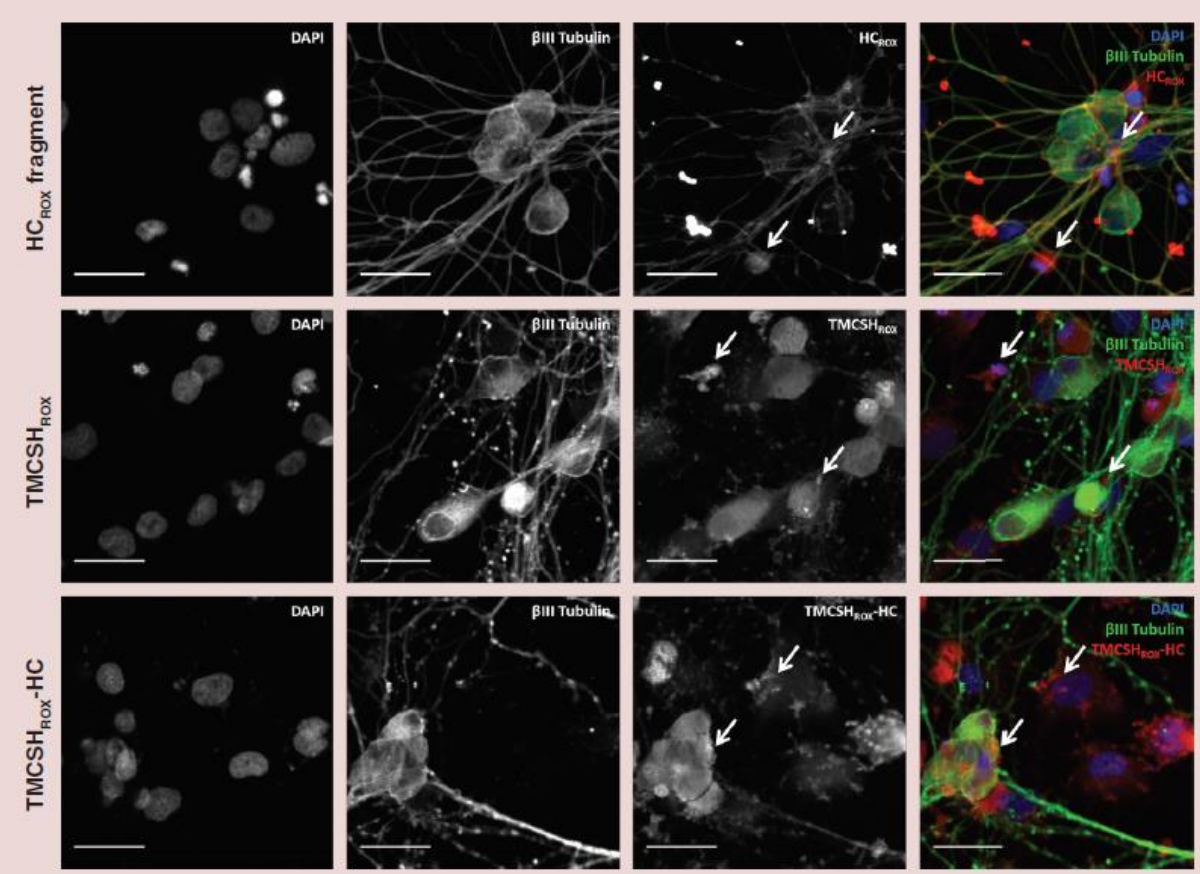

Figure 2. HC fragment and nanoparticle internalization in conventional dorsal root ganglia neuron cultures.

Representative images of dorsal root ganglia neurons cultured in 48-well plates (conventional cell cultures) and incubated with rhodamine-labeled HC fragment, $\mathrm{TMCSH}_{\mathrm{ROX}}$ or TMCSHROx-HC nanoparticles for $5 \mathrm{~h}$. For neuronal cells identification, cells were immunostained for $\beta$ III tubulin, a neuron-specific microtubule marker (green), and nuclei were stained with DAPI (blue). Rhodamine-labeled HC fragment and nanoparticles are identified in red (arrows). Scale bar: $25 \mu \mathrm{m}$.

DAPI: 4',6-diamidino-2-phenylindole; ROX: 5(6)-carboxy-X-rhodamine N-succinimidyl ester; TMCSH: Thiolated trimethyl chitosan 


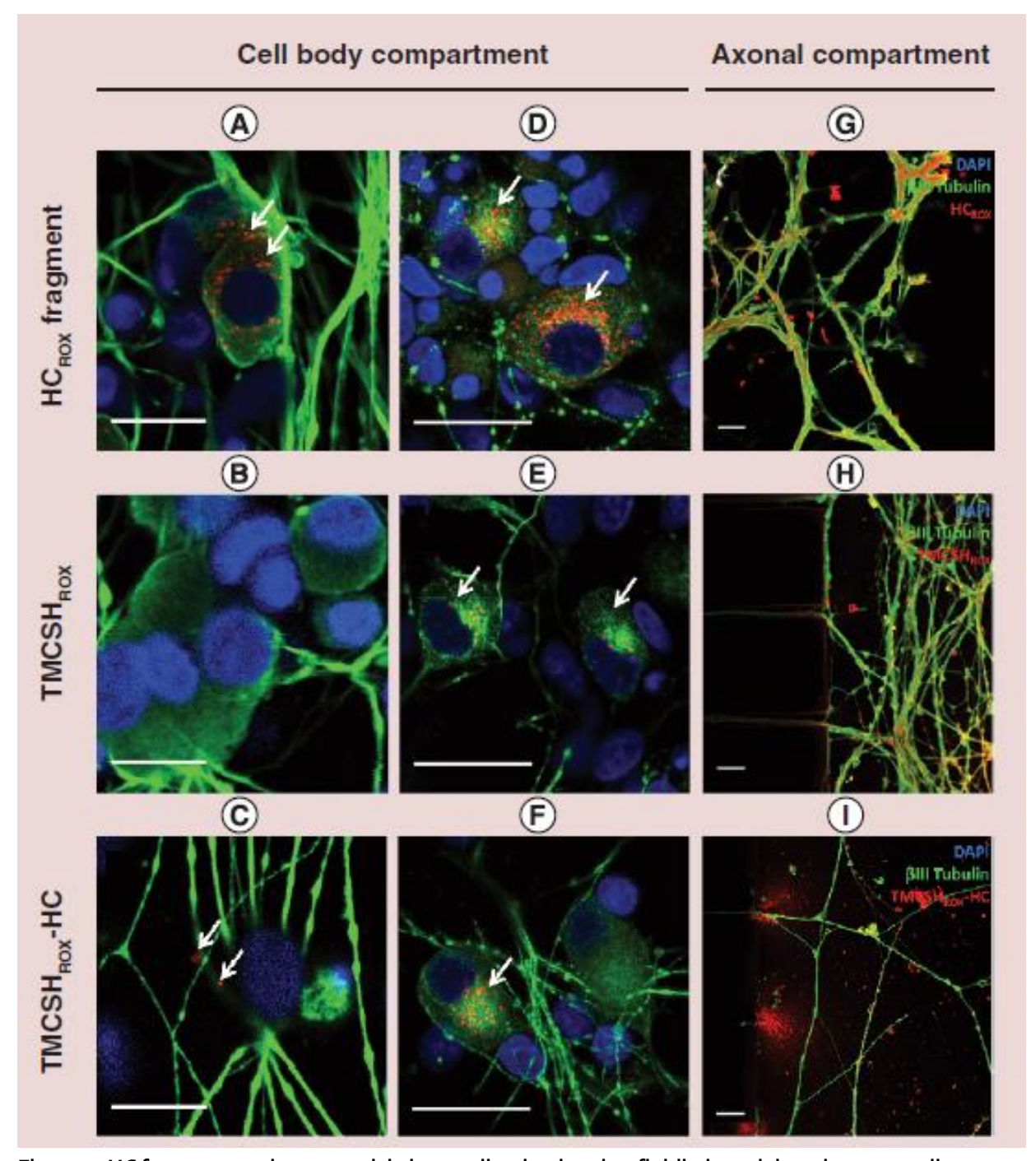

Figure 3. HC fragment and nanoparticle internalization in microfluidic-based dorsal root ganglia neuron cultures. Representative images of dorsal root ganglia neurons cultured in a microfluidic-based system and incubated with rhodamine-labeled HC fragment (A, D \& G), TMCSHROX (B, E \& H) or TMCSH $\mathrm{ROX}_{\text {R }}-\mathrm{HC}$ nanoparticles $(C, F \& I)$ for $5 \mathrm{~h}(\mathrm{~A}-\mathrm{C})$ or $12 \mathrm{~h}(\mathrm{D}-\mathrm{F})$ in the axonal compartment. For neuronal cells identification, cells were immunostained for $\beta$ III tubulin, a neuron-specific microtubule marker (green), and nuclei were stained with DAPI (blue). Rhodaminelabeled HC fragment and nanoparticles are identified in red (arrows). Scale bar: $25 \mu \mathrm{m}$.

ROX: 5(6)-carboxy-X-rhodamine N-succinimidyl ester; TMCSH: Thiolated trimethyl chitosan. 


\section{(A)}
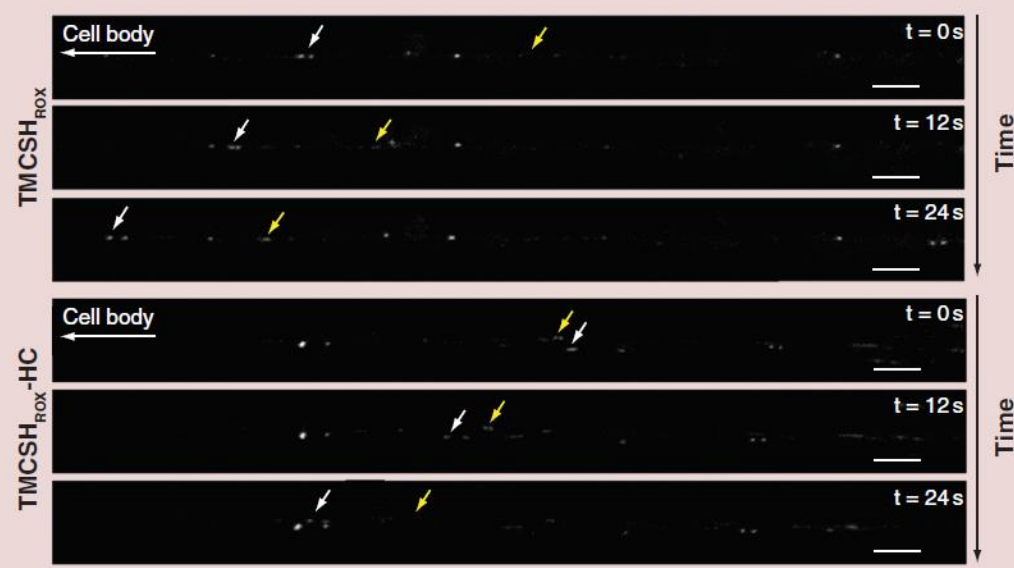

(B)

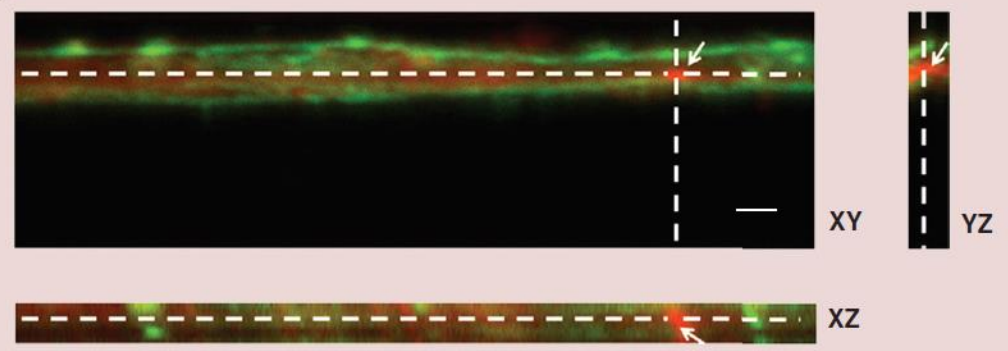

Figure 4. Retrograde axonal transport of thiolated trimethyl chitosan-based nanoparticles. (A) Application of nanoparticles to the axon terminals in the axonal compartment of microfluidic-based neuron cultures resulted in their retrograde axonal trafficking toward the cell body compartment. Scale bar: $5 \mu \mathrm{m}$. (B) Confocal image orthogonal views $(X Y, X Z, Y Z)$ through one axon in a microgroove showing a nanoparticle-loaded vesicle (arrow; red) being trafficked inside the axon, immunostained for $\beta$ III tubulin (green). Dashed lines indicate the selected region for the different planes of view. Scale bar: $1 \mu \mathrm{m}$. 


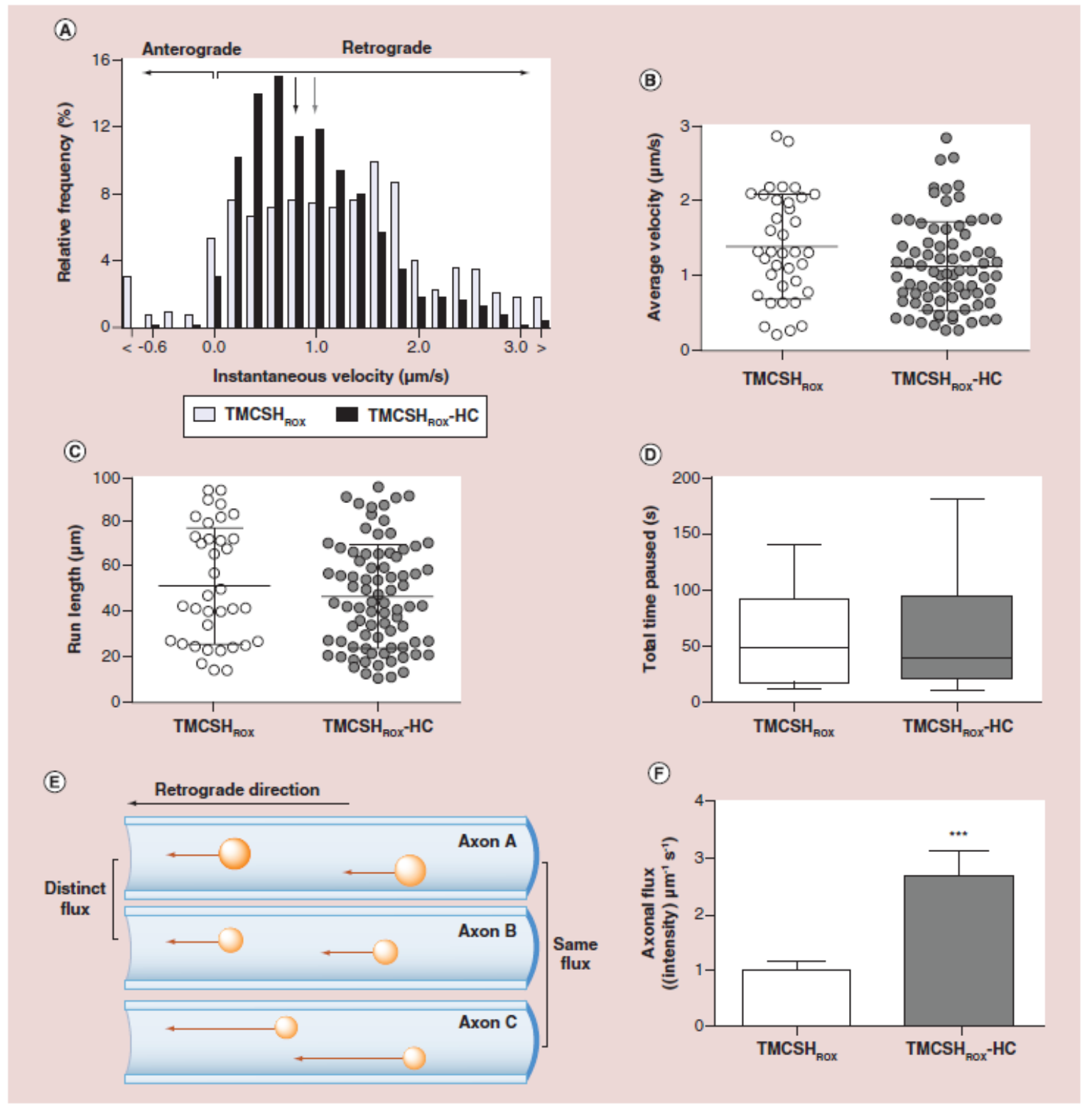

Figure 5. Retrograde axonal transport analysis of thiolated trimethyl chitosan-based nanoparticles. (A) Probability density function of the instantaneous velocity frequency of TMCSHROX and TMCSHROX-HC-loaded vesicles, defined as the speed $(\mu \mathrm{m} / \mathrm{s})$ of movement between two sequential images during a run. Each value represents the velocity of a single step between two frames. Arrows indicate the median value for instantaneous velocity distribution of each nanoparticle population. (B) Representation of the average velocity $(\mu \mathrm{m} / \mathrm{s})$ of retrograde axonal transport of nanoparticle-loaded vesicles, (C) total run length progressed ( $\mu \mathrm{m}$ ) and (D) total pause periods (s). Values represent mean $\pm S D$. (E) Illustration of longitudinal cross-section of three axons showing the retrograde transport of several vesicles and their mean velocity (represented by the arrows). The significance of measuring flux is depicted in the represented examples: vesicles traveling at the same mean velocity, but with different mean sizes, or in different numbers, generate distinct fluxes (axons A and B); or, vesicles traveling at different mean velocities and with different mean sizes may still give rise to identical fluxes (axons $A$ and $C$ ). (F)

INSTITUTO DE INVESTIGGAÇÃO E INOVAÇÃO EM SAÚDE UNIVERSIDADE DO PORTO

Rua Alfredo Allen, 208 4200-135 Porto Portugal +351 220408800 
Axonal flux ([intensity] $\mu \mathrm{m}-1 \mathrm{~s}-1$ ) of vesicles loaded with each nanoparticle type, normalized to the TMCSHROX flux value, and represented as mean \pm standard error of the mean.

$* * * p<0.001$

ROX: 5(6)-carboxy-X-rhodamine N-succinimidyl ester; SD: Standard deviation; TMCSH: Thiolated trimethyl chitosan.

(A)

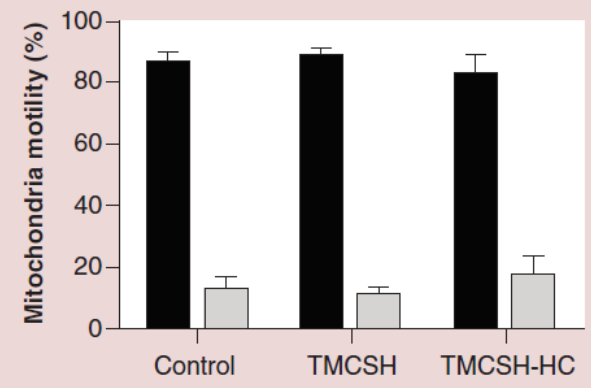

(B)

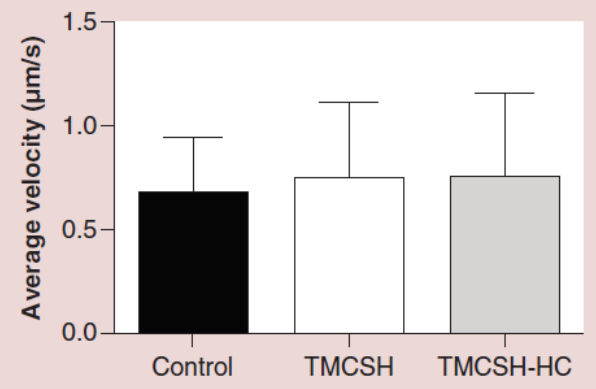

Stationary Moving

Figure 6. Mitochondria axonal transport characterization after nanoparticle treatment. (A) Characterization of mitochondria motility (\%) as paused/stationary or moving. (B) Average velocity $(\mu \mathrm{m} / \mathrm{s})$ of moving mitochondria. Values represent mean \pm SD.

SD: Standard deviation. 


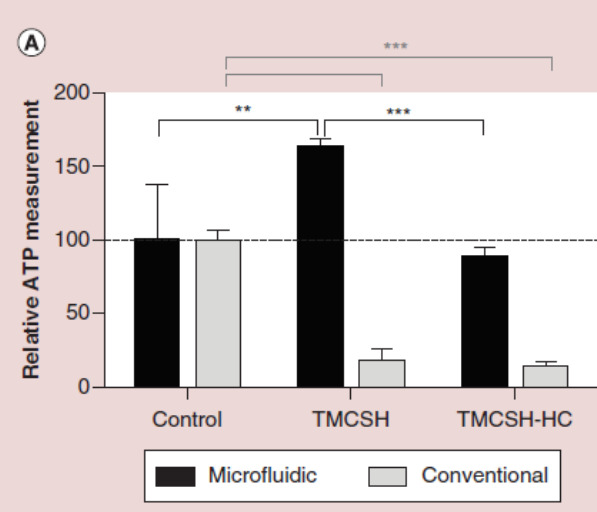

(C)

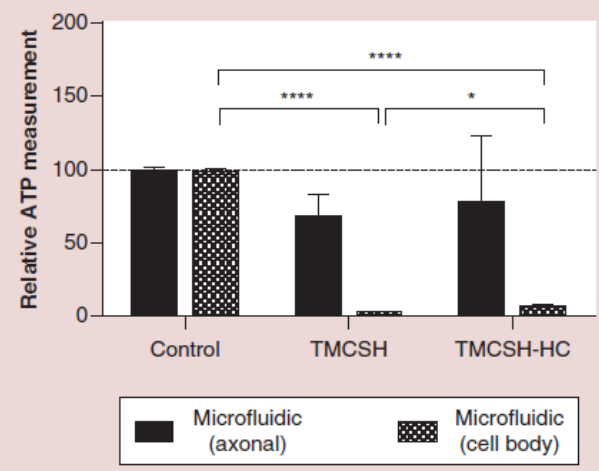

(B)

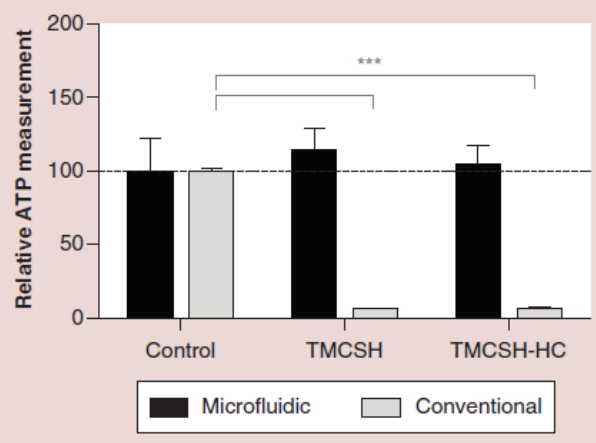

(D)

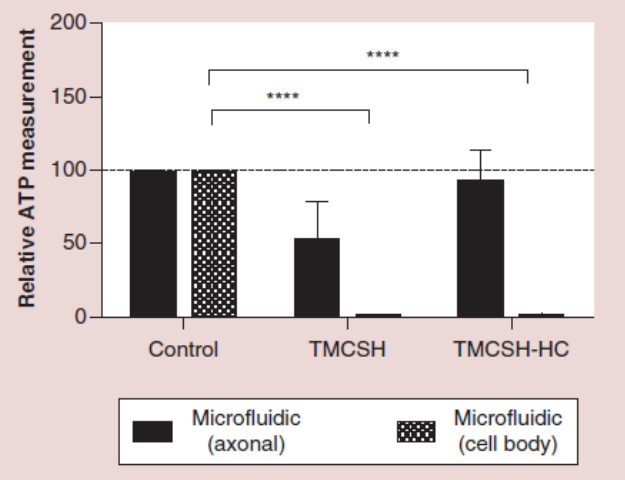

Figure 7. Impact of nanoparticle treatment in neuronal metabolic activity. (A \& B) Metabolic activity of DRG neurons after nanoparticle administration to the axonal terminals (microfluidic chambers) versus to the whole cells (96-wells plate, conventional cultures). (C \& D) Metabolic activity of DRG neurons cultured in microfluidic chambers after nanoparticle administration to the axonal terminals or cell body. Cellular ATP levels were measured after incubation with nanoparticles vehicle (control), TMCSH or TMCSHHC nanoparticles for 6 (A \& C) or $12 h(B \& D)$. The ATP levels are expressed as a percentage of control cultures. Mean $\pm S D ; n=3$ per condition.

$* \mathrm{p}<0.05 i^{* * \mathrm{p}}<0.01 ; * * * \mathrm{p}<0.001 ; * * * \mathrm{p}<0.0001$.

DRG: Dorsal root ganglia; SD: Standard deviation. 
Table 1. Physicochemical characterization of rhodamine-labeled thiolated trimethyl chitosan-based nanoparticles at an N/P ratio (moles of quaternized amine groups [N] to moles of DNA phosphate groups [P]) of 8 .

\begin{tabular}{|lcll|}
\hline Formulation & Size $(\mathrm{nm})$ & Pdi & $\zeta$ potential $(\mathrm{mV})$ \\
\hline $\mathrm{TMCSH}_{\mathrm{ROX}}$ & $273 \pm 4$ & $0.24 \pm 0.01$ & $+17.2 \pm 1.9$ \\
\hline TMCSH $_{\mathrm{ROX}}-\mathrm{HC}$ & $281 \pm 12$ & $0.25 \pm 0.01$ & $+16.4 \pm 2.5$ \\
\hline $\begin{array}{l}\text { Nanoparticles were characterized in terms of size, Pdi and } \zeta \text { potential. All values are representative of three independent experiments and are } \\
\text { expressed as mean } \pm \text { SD. } \\
\text { Pdi: Polydispersity index; ROX: } 5(6) \text {-carboxy-X-rhodamine N-succinimidyl ester; SD: Standard deviation; TMCSH: Thiolated trimethyl } \\
\text { chitosan. }\end{array}$ & \\
\hline
\end{tabular}

\section{Executive summary}

\section{Background}

- Nanoparticles have opened new exciting avenues for the development of therapeutic strategies for nervous system diseases. Consequently, the search for neuron-targeted nanoparticles as specific delivery vectors of therapeutic agents has increased, as well as efficient minimally invasive peripheral routes of administration.

- The currently available in vitro platforms and in vivo models to test nanoparticles in the framework of the nervous system have shown limitations.

\section{Methods}

- Compartmentalized dorsal root ganglia neuron cultures were established using microfluidic devices.

- Neuron-targeted and nontargeted nanoparticles based on thiolated trimethyl chitosan were prepared and incubated with neuron axonal terminals in the axonal compartment of microfluidic-based neuron cultures.

- The explored nanoparticle-targeting moiety was the nontoxic carboxylic terminal fragment from the tetanus neurotoxin $(\mathrm{HC})$.

- Nanoparticle bio-performance was evaluated by real-time visualization and quantification of the axonal transport of nanoparticle-loaded vesicles, as well as by neuronal metabolic activity analysis.

\section{Results \& discussion}

- Both nanoparticles were internalized at axon terminals and retrogradely transported to cell bodies at similar average velocities.

- Neuron-targeted nanoparticles showed a 2.7-times higher axonal flux than nontargeted nanoparticles, suggesting an improvement in cargo transportation efficiency to the cell bodies.

- Peripheral administration of nanoparticles to axon terminals is nontoxic as compared with their direct administration to the whole neuron (cell body and axons), the approach used in the conventional in vitro neuron cultures to test nanoparticles cytotoxic effect.

\section{Conclusion}

- The application of this microfluidic-based platform provided experimental evidence for the retrograde axonal transport and safety of the thiolated trimethyl chitosan-HC nanoparticles.

- This microfluidic-based neuron culture showed to be of added value in the evaluation of cell-nanoparticle interactions, nanoparticle axonal transport and safety, allowing for their rapid translation to subsequent in vivo studies. 


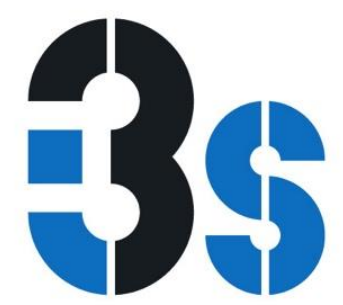

Version: Postprint (identical content as published paper) This is a self-archived document from i3S - Instituto de Investigação e Inovação em Saúde in the University of Porto Open Repository For Open Access to more of our

publications, please visit http://repositorio-aberto.up.pt/

NSTITUTO

DE INVESTIIGAÇÃO

EINOVAÇAO

EM SAÚDE

UNIVERSIDADE

DO PORTO

Rua Alfredo Allen, 208

4200-135 Porto

Portugal

+351 220408800

info@i3s.up.pt

www.i3s.up.pt 